\title{
Chemical Stability of Reconstituted Sincalide in Sterile Water Under 2 Different Storage Conditions
}

\author{
Eric Belanger, Shawn M. Wolf, Steven A. Zatt, Jeffrey Christoff, and Yousif Rojeab \\ Department of Pharmaceutical and Biomedical Sciences, Ohio Northern University, Ada, Ohio
}

This study aimed to evaluate the chemical stability of sincalide at 2 common storage conditions-room temperature and refrigeration-in an attempt to simulate the conditions faced during centralized reconstitution and subsequent distribution to regional clinical facilities. Sincalide is a peptide hormone product administered parenterally as an aid for diagnostic imaging of hepatobiliary conditions. With an estimated postreconstitution shelf-life of $8 \mathrm{~h}$ (updated by the manufacturer in 2014 with limited supporting data) and frequent shortages due to an intermittent supply, there is both clinical and economic value in the experimental determination of the true chemical stability of this agent. Methods: Sincalide was reconstituted and stored at both temperatures ( $n=4$ each), and samples were collected at predetermined time points. A validated high-performance liquid chromatography analytic method was used for quantification of the active ingredient in these samples. Results: Little to no chemical degradation of sincalide was observed for the duration of study, over $8 \mathrm{~d}$, after reconstitution and storage at room temperature. A trend toward a cyclic fluctuation in concentration was also shared among all samples. A similar trend toward little to no chemical degradation and cyclic pattern was observed for the duration of study, over $8 \mathrm{~d}$, after reconstitution and storage in refrigeration. Conclusion: This study supports that from a chemical standpoint, sincalide may potentially be used up to at least $8 \mathrm{~d}$ after reconstitution with sterile water, thus providing convenience and cost-saving benefits to medical institutions using the product. The findings of this study, however, warrant microbial testing over this storage duration before any recommendations for extended use can be made.

Key Words: sincalide; chemical stability; reconstitution; peptide stability

J Nucl Med Technol 2020; 48:174-176

DOI: 10.2967/jnmt.119.230441

\section{$\mathbf{S}$}

incalide is an 8-amino-acid, cholecystopancreaticgastrointestinal peptide hormone used as an aid to contract the gallbladder for diagnostic imaging purposes in hepatobiliary diseases (1). Sincalide for injection (Kinevac; Bracco

\footnotetext{
Received May 3, 2019; revision accepted Sep. 27, 2019.

For correspondence or reprints contact: Yousif Rojeab, Department of Pharmaceutical and Biomedical Sciences, Ohio Northern University, $525 \mathrm{~S}$. Main St., Ada, $\mathrm{OH} 45810$.

E-mail: y-rojeab@onu.edu

Published online Feb. 28, 2020.

COPYRIGHT (c) 2020 by the Society of Nuclear Medicine and Molecular Imaging.
}

Diagnostic Inc.) is supplied in a single-use, reconstitutable vial containing $5 \mu \mathrm{g}$ of sincalide. This agent is dosed at 0.02 or $0.04 \mu \mathrm{g} / \mathrm{kg}$ depending on the diagnostic procedure (1), resulting in an average dose of $1.4 \mu \mathrm{g}$ being administered to a prototypical $70-\mathrm{kg}$ patient. This leads to wastage of a significant portion of the vial, approximately $67 \%$, when administered to an average adult.

With an estimated postreconstitution shelf-life of $8 \mathrm{~h}$ (updated by the manufacturer in 2014 with limited supporting data) $(1,2)$ and frequent shortages due to an intermittent supply (Kim Giordano, Vice President and General Manager of Nuclear Medicine at Bracco Diagnostics Inc., written communication, May 23, 2014), there is both clinical and economic value in the experimental determination of the true chemical stability of this agent.

Littleton et al. reported a similar shelf-life for sincalide after $8 \mathrm{~h}$ after reconstitution: $80.05 \% \pm 4.07 \%$ and $89.73 \%$ $\pm 2.49 \%$ in normal saline and sterile water, respectively. However, that study did not evaluate the stability of sincalide past $8 \mathrm{~h}$ or under refrigeration, which is a common storage condition of reconstituted sincalide (3).

The chemical stability of similar agents has been studied. Octreotide, another 8-amino-acid peptide hormone, has a stated 24-h postreconstitution shelf-life per the manufacturer's package insert (4). The objective of this study was to determine the stability of sincalide in sterile water at both room temperature and refrigeration, and for an extended duration of several days after reconstitution.

\section{MATERIALS AND METHODS}

\section{Materials}

For this study, we used sincalide, sterile water for injection, $\left(\operatorname{Tyr}\left[\mathrm{SO}_{3} \mathrm{H}\right]^{27}\right.$ ) cholecystokinin fragment 26-33 amide, high-performance liquid chromatography (HPLC)-grade water, sodium monophosphate monobasic, sodium phosphate dibasic, and 1-propranol. Sincalide was purchased from Bracco Diagnostics and was the commercial product evaluated in this study. Sterile water for injection was purchased from Hospira, Inc., and was used in the reconstitution of commercial sincalide. Cholecystokinin fragment 26-33 amide was acquired through Sigma-Aldrich as pure sincalide peptides. These were then diluted to varying concentrations and used in the construction of calibration curves for HPLC analysis.

Water, HPLC-grade, was acquired from Avantor Performance Materials, LLC, and was used in the preparation of the mobile phase. Sodium phosphate monobasic was purchased from Sigma-Aldrich, 
and sodium phosphate dibasic was acquired from Fisher Chemical. Both were used in the preparation of phosphate buffer (in the mobile phase). 1-propanol was purchased from Beantown Chemical and was also used in the preparation of the mobile phase.

\section{Sample Collection}

At $t=0,8$ vials of commercial sincalide were reconstituted with sterile water and stored at either room temperature $\left(22.2^{\circ} \mathrm{C}\right.$; labeled as vials $1-4)$ or refrigeration $\left(2^{\circ} \mathrm{C}-8^{\circ} \mathrm{C}\right.$; labeled as vials $\left.5-8\right), n=4$ each. Room-temperature vials were stored in a drawer to ensure protection from light equivalent to that in a closed refrigerator. The drawer and refrigerator were opened only to retrieve the vials for sample collection, ensuring a controlled storage environment. At predetermined time points $(0,8,16,24,32,40,52$, $64,76,100,124,148,172$, and $196 \mathrm{~h}), 100-\mu \mathrm{L}$ samples were collected and stored at $-80^{\circ} \mathrm{C}$ until analyzed by HPLC. Tuberculin syringes (27-gauge needle) were used on self-sealing vial stoppers to draw samples. The vials were also stored in resealable plastic bags to circumvent any significant diluent loss due to evaporation.

\section{HPLC Method}

A reverse-phase HPLC assay for quantification of sincalide in collected samples was used and validated on the basis of the method of Littleton et al. with slight modification (3). The method consisted of ultraviolet detection at $220 \mathrm{~nm}$ and a Symmetry C18 column $(4.6 \times 150 \mathrm{~mm}$; Waters). The mobile phase consisted of a 4:1 ratio of $150 \mathrm{mM}$ phosphate buffer and 1-propanol at a flow rate of $0.5 \mathrm{~mL} / \mathrm{min}$. The injection volume was $50 \mu \mathrm{L}$.

\section{Preparation of Calibration Curves}

Using pure sincalide, intra- and interday calibration curves were constructed using the HPLC method. Each calibration curve consisted of 4 concentrations $(0.25,1,1.25$, and $2.5 \mu \mathrm{g} / \mathrm{mL})$ prepared through serial dilution. The mobile phase was used as the diluent. Intra- and interday variability in data was established. A straight-line equation of the general format $y=m x+b$ was generated from all calibration curves constructed and was subsequently used in data analyses. No data points were dropped.

\section{Data Analysis}

The calibration curve trendline equation was used to convert experimental absorbance peaks $(\mathrm{mcV})$ to concentration $(\mu \mathrm{g} / \mathrm{mL})$. All experimental data were then standardized to an assumption of a concentration of $1 \mu \mathrm{g} / \mathrm{mL}$ at $\mathrm{t}=0$, per label claim. These values were then converted to percentage of concentration remaining compared with $\mathrm{t}=0$. For all data, mean values were produced, along with $\mathrm{SD}$ and coefficient of variation CV (defined as $[\mathrm{SD} /$ mean $] \times 100 \%$ ).

\section{RESULTS}

\section{Calibration Curve}

The HPLC assay was validated with intraday $(n=3)$ and interday $(n=6)$ variability, expressed as $\mathrm{CV}$, of $0.89 \%$ and $2.39 \%$, respectively (Fig. 1 ).

Little to no chemical degradation of sincalide was observed for the duration of the study, over $8 \mathrm{~d}$, after reconstitution and storage at room temperature (vials 1-4). A trend toward a cyclic fluctuation in concentration was also shared among all samples (Figs. 2 and 3).

A similar trend toward little to no chemical degradation and cyclic pattern was observed for the duration of study, over $8 \mathrm{~d}$, after reconstitution and storage in refrigeration (vials 5-8; Figs. 3 and 4).

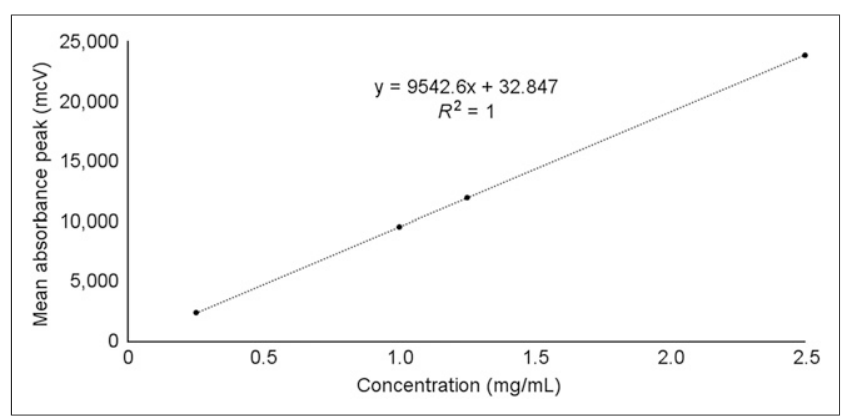

FIGURE 1. Mean data from absorbance peaks of pure sincalide dilutions were used to generate calibration curve and trendline equation.

\section{DISCUSSION}

The data collected suggest that sincalide in sterile water, at both room temperature and refrigeration, is chemically stable for at least $8 \mathrm{~d}$. Littleton et al. reported the stability of sincalide to be shorter; however, that study analyzed stability over only $8 \mathrm{~h}$ since the primary objective was to compare sincalide stability on reconstitution in normal saline versus sterile water (3). Octreotide, another 8-aminoacid peptide, has a shelf-life of $24 \mathrm{~h}$ after reconstitution per the manufacturer (4), which is supported by an independent accelerated stability study (5). As a similar molecule, it is plausible for sincalide to also demonstrate chemical stability beyond $8 \mathrm{~h}$.

Although the final outcome indicates significantly longer stability than the above sincalide study (3), the findings of this study demonstrate a similar and consistent pattern of decreased concentration by hour 8 (Figs. 2 and 3). After that time point, though, the concentration of sincalide increased and continued to fluctuate in a repetitive pattern until it appeared to reach equilibrium.

A possible explanation for this concentration-fluctuation pattern is a conformational change of sincalide in water. Sincalide may cycle through conformation changes while dissolved, thereby absorbing ultraviolet light in different capacities. This observation has not been explicitly reported

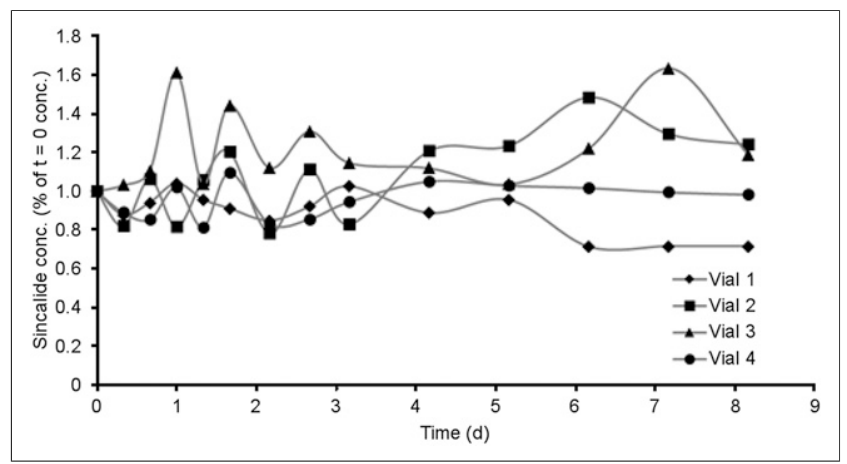

FIGURE 2. Percentage of concentration remaining at room temperature compared with that at $\mathrm{t}=0$ plotted against respective time points. 


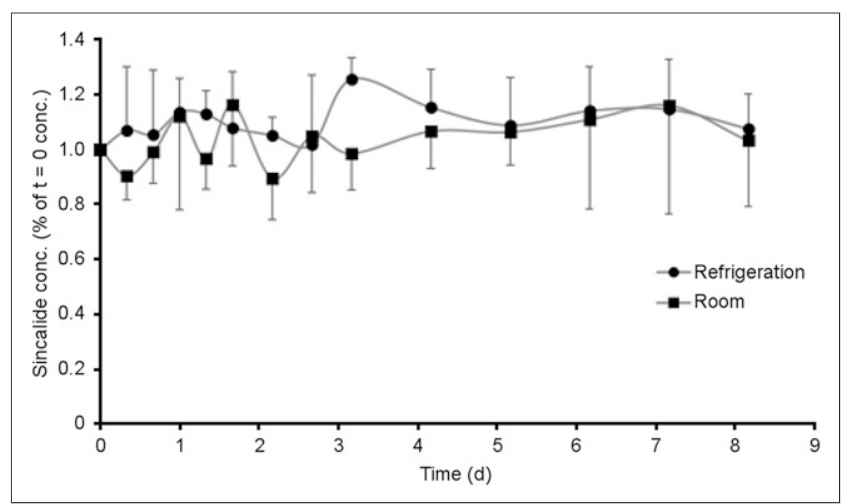

FIGURE 3. Mean values for percentage of concentration remaining at room and refrigerated temperatures, side by side, compared with that at $t=0$ plotted against respective time points. Error bars denote SD.

with similar molecules, as there are limited data on long-term stability evaluations of reconstituted peptides or proteins with frequent sampling as in this study. However, changes in the folding pattern, if they occur, would result in a change in ultraviolet absorbance as previously reported; folded proteins do exhibit changes in ultraviolet absorbance at $230 \mathrm{~nm}$, very similar to this study's absorbance of $220 \mathrm{~nm}(6)$.

Possible confounders were considered when observing and interpreting this data pattern. The drawer where roomtemperature vials were kept, and the refrigerator, were opened only to retrieve vials for sample collection, ensuring a controlled storage environment. This procedure limited the samples' exposure to light and temperature changes. Additionally, all samples collected were covered to prevent evaporation of reconstituted solution. Although temperature

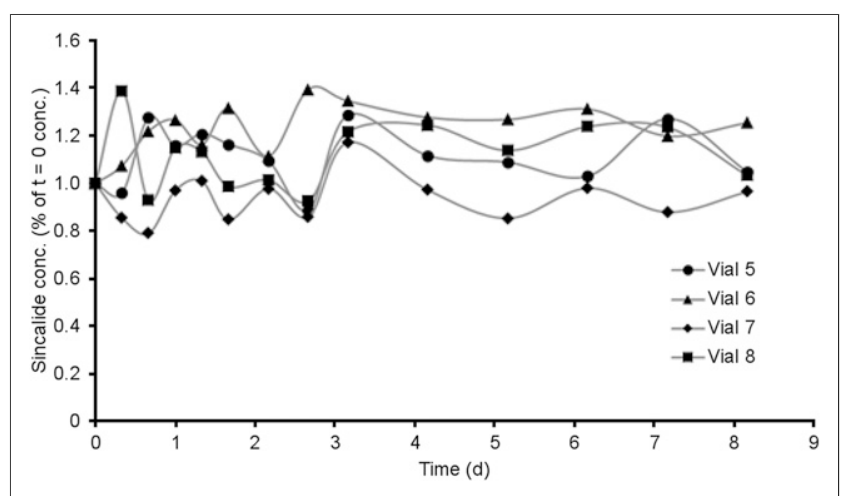

FIGURE 4. Percentage of concentration remaining at refrigerated temperature compared with that at $t=0$ plotted against respective time points. was not directly monitored at each point of sample collection, both the room and the refrigerator were kept at constant temperature settings and did not have a tendency to deviate.

Although this concentration-fluctuation pattern warrants further research to learn the mechanism behind it, this study's data support that sincalide is chemically stable for at least $8 \mathrm{~d}$ under both storage conditions evaluated. On the basis of these findings and of sincalide's being a drug with frequent supply-chain interruptions, one can conclude that prolonging the expiration time of sincalide may have a costsaving benefit, as less product is likely to be wasted. However, it is noteworthy that the objectives of this study did not include examination of bacterial growth or pyrogenicity on samples collected. Therefore, no recommendation on continued use of sincalide beyond $8 \mathrm{~h}$ after reconstitution is suggested, as U.S. Pharmacopeia guidelines state that any reconstituted medication should not be used past the expiration time specified by the manufacturer (7).

\section{CONCLUSION}

Results from this study demonstrated little to no chemical degradation of sincalide for its entire duration at both room temperature and refrigeration. These findings support that from a chemical standpoint, sincalide may potentially be used up to at least $8 \mathrm{~d}$ after reconstitution with sterile water, thus providing convenience and cost-saving benefits to medical institutions using the product. The findings of this study warrant microbial testing over this storage duration before any recommendations on extended usage can be made.

\section{DISCLOSURE}

Funding was provided by the Ohio Northern University Raabe College of Pharmacy. No other potential conflict of interest relevant to this article was reported.

\section{REFERENCES}

1. Kinevac ${ }^{\circledR}$ sincalide for injection [package insert]. Monroe Township, NJ: Bracco Diagnostics; July 2014

2. Kinevac ${ }^{\circledR}$ sincalide for injection [package insert]. Princeton, NJ: Bracco Diagnostics; November 1994.

3. Littleton LT, Fileta BB, Massey R, Wood TI. Kinevac stability after reconstitution with sodium chloride injection USP, 0.9\%. J Nucl Med Technol. 2009;37:57-59.

4. Sandostatin ${ }^{\circledR}$ octreotide acetate injection [package insert]. East Hanover, NJ: Novartis Pharmaceuticals Corporation; June 2018.

5. Na DH, DeLuca PP. PEGylation of octreotide: I. separation of positional isomers and stability against acylation by poly(D,L-lactide-co-glycolide). Pharm Res. 2005;22: 736-742.

6. Liu PF, Avramova LV, Park C. Revisiting absorbance at $230 \mathrm{~nm}$ as a protein unfolding probe. Anal Biochem. 2009;389:165-170.

7. pharmaceutical compounding: sterile preparations. In: United States Pharmacopeia. Rockville, MD: United States Pharmacopeial Convention; 2016. 